PROCEEDINGS OF THE

AMERICAN MATHEMATICAL SOCIETY

Volume 136, Number 7, July 2008, Pages 2365-2373

S 0002-9939(08)09270-8

Article electronically published on March 11, 2008

\title{
TOPOLOGICAL RADON TRANSFORMS AND DEGREE FORMULAS FOR DUAL VARIETIES
}

\author{
YUTAKA MATSUI AND KIYOSHI TAKEUCHI \\ (Communicated by Ted Chinburg)
}

\begin{abstract}
We give a simpler and purely topological proof of Ernström's class formula (1997) for the degree of dual varieties. Our new proof also allows us to obtain a formula describing the degrees of the associated varieties studied by Gelfand, Kapranov and Zelevinsky (1994).
\end{abstract}

\section{INTRODUCTION}

The study of projective duality is a very rich subject in algebraic geometry. Let $V \subset \mathbb{P}_{n}$ be a projective variety over $\mathbb{C}$. The dual $V^{*}$ of $V$ is usually a hypersurface in the dual projective space $\mathbb{P}_{n}^{*}$, and it is a natural problem to determine the degree of the hypersurface $V^{*}$. Many mathematicians have obtained formulas for the degree of $V^{*}$, which is called the class of $V$. For example, assuming that $V$ is smooth, Kleiman [10] proved a formula which expresses $\operatorname{deg} V^{*}$ in terms of the Chern classes of a vector bundle on $V$. In this situation, an algorithm, called Cayley's method, to compute the defining polynomial of $V^{*}$ was also established by GelfandKapranov-Zelevinsky in [6]. For other useful class formulas, see the excellent survey by Tevelev [18. However to the best of our knowledge, if the projective variety $V$ is singular, only the class formula in Ernström [4] is applicable. In his formula, $\operatorname{deg} V^{*}$ is described by the topological integrals of the Euler obstruction of $V$. Ernström 4 proved this formula by combining some classical deep results in enumerative algebraic geometry, which heavily depend on the specific situation. The aim of this paper is to give a simpler and purely topological proof of Ernström's important result in [4]. We show that this class formula can be easily obtained from a result in his other paper [3] (see also [13, for a new proof and its generalizations) just by applying some elementary lemmas on constructible functions (Lemmas 2.4 and 2.6). Moreover this new proof allows us to extend Ernström's class formula to various situations. In particular, we apply our method to the study of the $k$-th associated variety $V^{\langle k\rangle}(0 \leq k \leq n-1)$ studied by Gelfand-Kapranov-Zelevinsky [6] and Ernström [3].

Recall that if $V \subset \mathbb{P}_{n}$ is smooth, $V^{\langle k\rangle}$ is the subvariety of the Grassmann manifold $\mathbb{G}_{n, k}$ consisting of $k$-dimensional (linear) projective subspaces $\widehat{L}$ of $\mathbb{P}_{n}$ such that there exists a point $x \in V \cap \widehat{L}$ at which $\widehat{L}$ does not intersect $V$ transversally.

Received by the editors September 13, 2005, and, in revised form, November 16, 2006, March 7, 2007, and May 7, 2007.

2000 Mathematics Subject Classification. Primary 14B05, 14N99, 32C38, 35A27, 53A20.

(C)2008 American Mathematical Society 
According to Chapter 3 of [6], if $V^{\langle k\rangle}$ is a hypersurface in the Grassmann manifold $\mathbb{G}_{n, k}$, we can define the degree of $V^{\langle k\rangle}$ to be that of its defining polynomial in the Plücker coordinates: $V^{\langle k\rangle} \subset \mathbb{G}_{n, k} \subset \mathbb{P}_{\left(\begin{array}{c}n+1 \\ k+1\end{array}\right)-1}$. In this setting, our formula in Theorem 3.4 gives an expression of $\operatorname{deg} V^{\langle k\rangle}$ using the topological integrals of the Euler obstruction of $V$. Note that in a forthcoming paper [15], the condition on $V$ for the $k$-th associated variety $V^{\langle k\rangle}$ to be a hypersurface will be precisely studied. Finally, let us mention that results obtained in this paper were effectively used in [14] to deduce various concrete class formulas.

\section{Preliminary notions and Results}

In this section, we briefly recall the theory of constructible functions and dual varieties. We also give some lemmas which are useful in studying topological Radon transforms.

The main reference on constructible functions is [9, Chapter IX] by KashiwaraSchapira, and we follow the terminology in this reference throughout this paper. For example, for a topological space $X$, we denote by $\mathbf{D}_{\mathbb{R}-c}^{b}(X)$ the derived category of bounded complexes of sheaves of $\mathbb{C}_{X}$-modules whose cohomologies are $\mathbb{R}$-constructible.

2.1. Constructible functions. Let $X$ be a real analytic manifold.

Definition 2.1. We say that a $\mathbb{Z}$-valued function $\varphi: X \longrightarrow \mathbb{Z}$ is constructible if there exists a locally finite family of compact subanalytic subsets $\left\{K_{\alpha}\right\}_{\alpha \in A}$ such that

$$
\varphi=\sum_{\alpha \in A} m_{\alpha} \mathbf{1}_{K_{\alpha}}
$$

where $m_{\alpha} \in \mathbb{Z}$ and for a subset $Z \subset X$ we denote by $\mathbf{1}_{Z}$ the characteristic function of $Z$.

We set $C F(X)=\{\varphi: X \longrightarrow \mathbb{Z} \mid \varphi$ is constructible $\}$.

For $F \in \mathbf{D}_{\mathbb{R}-c}^{b}(X)$, the function $\chi(F): X \longrightarrow \mathbb{Z}$ defined by taking the local Euler-Poincaré index

$$
\chi(F)(x)=\sum_{j \in \mathbb{Z}}(-1)^{j} \operatorname{dim} H^{j}\left(F_{x}\right)
$$

at each $x \in X$ is constructible.

Let us define the Grothendieck group $\mathbf{K}_{\mathbb{R}-c}(X)$ of $\mathbf{D}_{\mathbb{R}-c}^{b}(X)$ to be the quotient of the free abelian group generated by the objects in $\mathbf{D}_{\mathbb{R}-c}^{b}(X)$ by the relations

$$
F=F^{\prime}+F^{\prime \prime} \quad\left(F^{\prime} \rightarrow F \rightarrow F^{\prime \prime} \stackrel{+1}{\rightarrow} \text { is a distinguished triangle }\right) .
$$

By the property

$$
F^{\prime} \rightarrow F \rightarrow F^{\prime \prime} \stackrel{+1}{\rightarrow} \quad \text { is a distinguished triangle } \quad \Longrightarrow \quad \chi(F)=\chi\left(F^{\prime}\right)+\chi\left(F^{\prime \prime}\right)
$$

of the local Euler-Poincaré index $\chi$, we obtain a group homomorphism

$$
\chi: \mathbf{K}_{\mathbb{R}-c}(X) \longrightarrow C F(X) .
$$

Theorem 2.2 ([9, Theorem 9.7.1]) $\chi$ is an isomorphism. 
We recall basic operations of constructible functions. These operations are induced by those of constructible sheaves through this Euler-Poincaré index $\chi$.

Definition 2.3. Let $X$ and $Y$ be real analytic manifolds and $f: X \longrightarrow Y$ a real analytic map.

(i) For a constructible function $\varphi \in C F(X)$ with compact support, we define the Euler (topological) integral of $\varphi$ by

$$
\int_{X} \varphi=\chi(X ; F) \quad(=\chi(R \Gamma(X ; F))) \quad \in \mathbb{Z},
$$

where $F \in \mathbf{K}_{\mathbb{R}-c}(X)$ is given by $\chi(F)=\varphi$. This Euler integral $\int_{X} \varphi$ can be calculated more easily as follows. If $\varphi=\sum_{\alpha \in A} m_{\alpha} \mathbf{1}_{K_{\alpha}}$ for a family of finite compact subanalytic subsets $\left\{K_{\alpha}\right\}_{\alpha \in A}$ as in Definition 2.1, then we have

$$
\int_{X} \varphi=\sum_{\alpha \in A} m_{\alpha} \chi\left(K_{\alpha}\right)
$$

(ii) Let $\varphi \in C F(X)$. Assume that $f: \operatorname{supp}(\varphi) \longrightarrow Y$ is proper. We define the direct image $\int_{f} \varphi \in C F(Y)$ of $\varphi$ by

$$
\left(\int_{f} \varphi\right)(y)=\int_{X} \varphi \cdot \mathbf{1}_{f^{-1}(y)}
$$

Note that if moreover $X$ is compact, the following diagram is commutative:

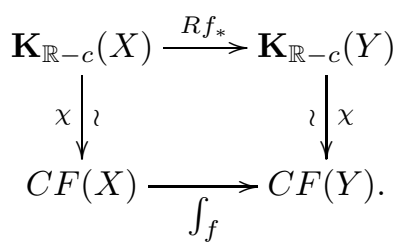

(iii) Let $\psi \in C F(Y)$. We define the inverse image $f^{*} \psi \in C F(X)$ of $\psi$ by

$$
\left(f^{*} \psi\right)(x)=\psi(f(x)) .
$$

Clearly this operation is also compatible with the one $f^{-1}: \mathbf{K}_{\mathbb{R}-c}(Y) \longrightarrow$ $\mathbf{K}_{\mathbb{R}-c}(X)$ for constructible sheaves.

Lemma 2.4. Let $f: X \longrightarrow Y$ be a proper map of real analytic manifolds, $\varphi \in$ $C F(X)$ and $\psi \in C F(Y)$. Then we have

$$
\left(\int_{f} \varphi\right) \cdot \psi=\int_{f}\left(\varphi \cdot f^{*} \psi\right)
$$

Proof. We take constructible sheaves $F \in \mathbf{D}_{\mathbb{R}-c}^{b}(X), G \in \mathbf{D}_{\mathbb{R}-c}^{b}(Y)$ satisfying $\chi(F)=\varphi$ and $\chi(G)=\psi$. Then by the projection formula for sheaves

$$
R f_{*}\left(F \stackrel{L}{\otimes} f^{-1} G\right) \simeq\left(R f_{*} F\right) \stackrel{L}{\otimes} G,
$$

we have

$$
\begin{aligned}
\left(\int_{f} \varphi\right) \cdot \psi & =\chi\left(R f_{*} F\right) \cdot \chi(G)=\chi\left(\left(R f_{*} F\right) \stackrel{L}{\otimes} G\right)=\chi\left(R f_{*}\left(F \stackrel{L}{\otimes} f^{-1} G\right)\right) \\
(2.13) & =\int_{f} \chi(F) \cdot \chi\left(f^{-1} G\right)=\int_{f} \varphi \cdot f^{*} \psi .
\end{aligned}
$$


2.2. Euler obstructions. Let $X$ be an $n$-dimensional smooth algebraic variety over $\mathbb{C}$ and $V \subset X$ an algebraic subvariety in it. Following the definition of Kashiwara [8], let us introduce the Euler obstruction $\operatorname{Eu}_{V} \in C F(X)$ of $V$. This constructible function is zero outside $V$ and takes the value 1 on the smooth part $V_{\text {reg }}$ of $V$. Let $V=\bigsqcup_{\alpha} V_{\alpha}$ be a Whitney stratification of $V$ consisting of connected strata. We define the values $e_{\alpha}$ of the function $\mathrm{Eu}_{V}$ on the strata $V_{\alpha}$ by induction on the codimensions of the $V_{\alpha}$ in $V$ as follows:

(i) If $\operatorname{codim}_{V} V_{\alpha}=0$, then we set $e_{\alpha}=1$.

(ii) Suppose that $k=\operatorname{codim}_{V} V_{\alpha} \geq 1$ and that the values $e_{\beta}$ on the strata $V_{\beta}$ satisfying $\operatorname{codim}_{V} V_{\beta}<k$ have already been defined. Then the value $e_{\alpha}$ is defined by the following procedure. First take a point $x_{0} \in V_{\alpha}$ and choose a local coordinate system of $X$ in which $x_{0}=0$ and $V_{\alpha}=\left\{\left(x^{\prime}, x^{\prime \prime}\right) \in\right.$ $\left.\mathbb{C}^{d} \times \mathbb{C}^{n-d} \mid x^{\prime}=0\right\}\left(x^{\prime}=\left(x_{1}, \ldots, x_{d}\right), d=\operatorname{codim}_{X} V_{\alpha}\right)$. For a small enough $r>0$, consider the open ball

$$
B_{r}=\left\{x \in \mathbb{C}^{n}|| x \mid<r\right\} .
$$

Also for a conormal vector $p=\left(0 ;\left(\xi_{0}^{\prime}, 0\right)\right) \in T_{V_{\alpha}}^{*} \mathbb{C}^{n} \backslash \bigsqcup_{\beta \neq \alpha} \overline{T_{V_{\beta}}^{*} \mathbb{C}^{n}}$, set

$$
G_{p}=\left\{\left(x^{\prime}, x^{\prime \prime}\right) \in \mathbb{C}^{d} \times \mathbb{C}^{n-d} \mid \operatorname{Re}\left\langle x^{\prime}, \xi_{0}^{\prime}\right\rangle>0\right\} .
$$

Then we have

$$
e_{\alpha}=\operatorname{Eu}_{V}\left(x_{0}\right)=\sum_{\beta \neq \alpha, x_{0} \in \overline{V_{\beta}}} e_{\beta} \cdot \chi\left(B_{r} \cap G_{p} \cap V_{\beta}\right) .
$$

Note that this definition of $\mathrm{Eu}_{V} \in C F(X)$ is independent of the choice of the stratification $V=\bigsqcup_{\alpha} V_{\alpha}$ of $V$, the point $x_{0} \in V_{\alpha}$ and the covector $p$, etc. In particular, the function $\mathrm{Eu}_{V}$ is constant on each stratum $V_{\alpha}$.

2.3. Radon transforms of constructible functions. Let $X, Y$ and $S$ be real analytic manifolds. We consider the diagram:

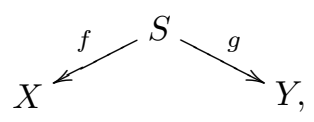

where $f$ and $g$ are proper morphisms.

Definition 2.5. We define the topological Radon transform of constructible functions and its dual transform by

$$
\begin{array}{ll}
\mathcal{R}_{S}=\int_{g} \circ f^{*}: & C F(X) \longrightarrow C F(Y), \\
\mathcal{R}_{S}^{\vee}=\int_{f} \circ g^{*}: & C F(Y) \longrightarrow C F(X) .
\end{array}
$$

In this general situation, we have the following lemma.

Lemma 2.6. Consider the diagram (2.17). If we assume moreover that $X$ and $Y$ are compact, then we have

$$
\int_{Y} \mathcal{R}_{S}(\varphi) \cdot \psi=\int_{X} \varphi \cdot \mathcal{R}_{S}^{\vee}(\psi)
$$

for any $\varphi \in C F(X)$ and $\psi \in C F(Y)$. 
Proof. Let $a_{X}: X \longrightarrow\{\mathrm{pt}\}$ and $a_{Y}: Y \longrightarrow\{\mathrm{pt}\}$ be the maps to a point. Then by Lemma 2.4 we have

$$
\begin{aligned}
\int_{Y} \mathcal{R}_{S}(\varphi) \cdot \psi & =\int_{a_{Y}}\left(\int_{g} f^{*} \varphi\right) \cdot \psi=\int_{a_{Y}} \int_{g}\left(f^{*} \varphi \cdot g^{*} \psi\right)=\int_{a_{Y} \circ g} f^{*} \varphi \cdot g^{*} \psi \\
(2.21) & =\int_{a_{X} \circ f} f^{*} \varphi \cdot g^{*} \psi=\int_{a_{X}} \varphi \cdot\left(\int_{f} g^{*} \psi\right)=\int_{X} \varphi \cdot \mathcal{R}_{S}^{\vee}(\psi) .
\end{aligned}
$$

2.4. Dual varieties. We define the Grassmann manifold $\mathbb{G}_{n, k}(1 \leq k \leq n-1)$ by

$$
\mathbb{G}_{n, k}=\left\{L \subset \mathbb{C}^{n+1} \mid L \text { is a }(k+1) \text {-dimensional linear subspace in } \mathbb{C}^{n+1}\right\} .
$$

For a $(k+1)$-dimensional vector space $L \in \mathbb{G}_{n, k}$, we denote its projectivization by $\widehat{L}$. This $\widehat{L}$ is a $k$-dimensional linear projective subspace of $\mathbb{P}_{n}=\mathbb{G}_{n, 0}$. In this paper, we identify the set of $k$-dimensional linear subspaces of $\mathbb{P}_{n}$ with $\mathbb{G}_{n, k}$.

Definition 2.7. Let $V$ be a subvariety of the $n$-dimensional projective space $\mathbb{P}_{n}$. We define the $k$-dual variety $V^{\langle k\rangle} \subset \mathbb{G}_{n, k}$ of $V$ to be the closure of the set of all $k$-dimensional linear subspaces $\widehat{L} \in \mathbb{G}_{n, k}$ such that there exists a point $x \in V_{\text {reg }} \cap \widehat{L}$ at which $\widehat{L}$ does not intersect with $V$ transversally.

In the case where $k=n-1$, the $k$-dual $V^{\langle k\rangle} \subset \mathbb{G}_{n, k} \simeq \mathbb{P}_{n}^{*}$ is the classical dual variety of $V$, which is usually denoted by $V^{*}$. In [6], Gelfand-Kapranov-Zelevinsky call $V^{\langle k\rangle}$ the $k$-th associated variety of $V$.

We consider the diagram (2.17) for $X=\mathbb{P}_{n}, Y=\mathbb{G}_{n, k}$ and

$$
S=\left\{(l, L) \in X \times Y \mid 0 \in l \subset L \subset \mathbb{C}^{n+1}\right\}
$$

(the incidence submanifold). Let $f$ and $g$ be the natural projections from $S$ to $X$ and $Y$ respectively. In this situation, the topological Radon transform (2.18) was studied by many mathematicians (see, for example, [2, 3], 12, 17] and 19, etc.). In particular, the following beautiful relation between the topological Radon transform $\mathcal{R}_{S}$ and dual varieties was established by Ernström [3].

Theorem 2.8 ([3, Theorem 3.2]). Let $V \subset X=\mathbb{P}_{n}$ be a projective variety. Then we have

$$
\mathcal{R}_{S}\left(\operatorname{Eu}_{V}\right)=(-1)^{k(n-k)+\operatorname{dim} V+\operatorname{dim} V^{\langle k\rangle}} \operatorname{Eu}_{V^{\langle k\rangle}}+e^{\langle k\rangle} \mathbf{1}_{\mathbb{G}_{n, k}},
$$

where $e^{\langle k\rangle}$ is the generic value of $\mathcal{R}_{S}\left(\mathrm{Eu}_{V}\right) \in C F(Y)$.

We remark that in the real case a similar result was obtained in 13 .

\section{Degrees of Dual Varieties}

In this section, we apply the results in Section 2 to some classical subjects in algebraic geometry. In particular, for a projective variety $V \subset \mathbb{P}_{n}$ over $\mathbb{C}$, we give a new proof of Ernström's formula which expresses the degree of the dual $V^{*} \subset \mathbb{P}_{n}^{*}$ in terms of the topology of $V$ (see [4). This new proof allows us to generalize Ernström's result.

First, let us consider the case where $X=\mathbb{P}_{n}$ and $Y=\mathbb{P}_{n}^{*}=\mathbb{G}_{n, n-1}$. For a projective variety $V \subset X$ of dimension $d$, we shall describe the degree of the dual $V^{*} \subset Y$ using the Euler obstruction $\operatorname{Eu}_{V} \in C F(X)$ of $V$. For this purpose, recall that the degree of $V^{*} \subset Y=\mathbb{P}_{n}^{*}$ is equal to its intersection number $\sharp\left(\widehat{L} \cap V^{*}\right)$ with a generic linear subspace $\widehat{L} \subset \mathbb{P}_{n}^{*}$ of the complementary dimension $n-\operatorname{dim} V^{*}$. Since 
the dual variety $V^{*}$ is usually a hypersurface in $Y=\mathbb{P}_{n}^{*}$, we set $r=(n-1)-\operatorname{dim} V^{*}$ and call it the dual defect of $V$. Hence the dimension of the linear subspace $\widehat{L} \subset$ $\mathbb{P}_{n}^{*}$ that we take is $r+1: \widehat{L} \simeq \mathbb{P}_{r+1}$. The following theorem slightly generalizes Ernström's result in [4].

Theorem 3.1. Let $\widehat{L} \subset Y=\mathbb{P}_{n}^{*}$ be a linear subspace of dimension $r+1$ such that $\widehat{L} \cap V_{\text {sing }}^{*}=\emptyset\left(V_{\text {sing }}^{*}=V^{*} \backslash V_{\text {reg }}^{*}\right.$ is the set of singular points in $\left.V^{*}\right)$. Regarding $\widehat{L}$ as a linear system in $X=\mathbb{P}_{n}$, consider its base locus $B_{S}(\widehat{L}) \simeq \mathbb{P}_{n-r-2} \subset X=\mathbb{P}_{n}$ defined by

$$
B_{S}(\widehat{L})=\bigcap_{[y] \in \widehat{L}}\{\widehat{y=0}\}
$$

where $[y]=\left[y_{0}: y_{1}: \cdots: y_{n}\right]$ is the homogeneous coordinate in the dual projective space $Y \simeq \mathbb{P}_{n}^{*}$. Then for a generic hyperplane $\widehat{H} \subset X=\mathbb{P}_{n}$ we have

$$
\chi\left(V^{*} \cap \widehat{L}\right)=(-1)^{d+r}\left\{(r+1) \int_{X} \mathrm{Eu}_{V}-(r+2) \int_{\widehat{H}} \mathrm{Eu}_{V}+\int_{B_{S}(\widehat{L})} \mathrm{Eu}_{V}\right\} .
$$

Proof. By Theorem 2.8, we have

$$
\mathcal{R}_{S}\left(\mathrm{Eu}_{V}\right)=\left(\int_{\widehat{H}} \mathrm{Eu}_{V}\right) \mathbf{1}_{Y}+(-1)^{d+r} \mathrm{Eu}_{V^{*}} .
$$

Then it follows from our assumption $\widehat{L} \cap V_{\text {sing }}^{*}=\emptyset$ that we have

$$
\int_{Y} \mathcal{R}_{S}\left(\mathrm{Eu}_{V}\right) \cdot \mathbf{1}_{\widehat{L}}=(r+2) \int_{\widehat{H}} \operatorname{Eu}_{V}+(-1)^{d+r} \chi\left(V^{*} \cap \widehat{L}\right) .
$$

Since by Lemma 2.6 we have

$$
\int_{Y} \mathcal{R}_{S}\left(\mathrm{Eu}_{V}\right) \cdot \mathbf{1}_{\widehat{L}}=\int_{X} \operatorname{Eu}_{V} \cdot \mathcal{R}_{S}^{\vee}\left(\mathbf{1}_{\widehat{L}}\right)=\int_{X} \operatorname{Eu}_{V} \cdot\left\{(r+1) \mathbf{1}_{X}+\mathbf{1}_{B_{S}(\widehat{L})}\right\},
$$

we finally obtain

$$
(-1)^{d+r} \chi\left(V^{*} \cap \widehat{L}\right)=(r+1) \int_{X} \operatorname{Eu}_{V}-(r+2) \int_{\widehat{H}} \mathrm{Eu}_{V}+\int_{B_{S}(\widehat{L})} \mathrm{Eu}_{V} .
$$

This completes the proof.

If we assume moreover that $\widehat{L}$ intersects with $V^{*}$ transversally, we recover the following important result proved first by Ernström [4] in a completely different way.

Corollary $3.2([4])$. Let $\widehat{H} \subset X=\mathbb{P}_{n}$ (resp. $\widehat{H_{r+2}} \subset X=\mathbb{P}_{n}$ ) be a generic hyperplane (resp. a generic linear subspace of codimension $r+2)$. Then the degree of the dual $V^{*} \subset Y=\mathbb{P}_{n}^{*}$ is given by

$$
(-1)^{d+r}\left\{(r+1) \int_{X} \mathrm{Eu}_{V}-(r+2) \int_{\widehat{H}} \mathrm{Eu}_{V}+\int_{\widehat{H_{r+2}}} \mathrm{Eu}_{V}\right\} .
$$

Remark 3.3. By the proof of Corollary 3.2 we can also determine the dimension of the dual variety $V^{*}$ as follows. For each non-negative integer $i \geq 0$, take a generic 
hyperplane $\widehat{H} \subset X=\mathbb{P}_{n}$ (resp. a generic linear subspace $\widehat{H_{i+2}} \subset X=\mathbb{P}_{n}$ of codimension $i+2)$ and set

$$
\delta_{i}:=(-1)^{d+i}\left\{(i+1) \int_{X} \mathrm{Eu}_{V}-(i+2) \int_{\widehat{H}} \mathrm{Eu}_{V}+\int_{\widehat{H_{i+2}}} \mathrm{Eu}_{V}\right\} .
$$

Then for any $i<r(r$ is the dual defect of $V)$, generic linear subspaces $\widehat{L} \subset Y=\mathbb{P}_{n}^{*}$ of dimension $i+1$ in $Y=\mathbb{P}_{n}^{*}$ do not meet with $V^{*}$ and $\delta_{i}=0$. Hence the dual defect $r$ of $V$ is characterized as the non-negative integer $k \geq 0$ such that $\delta_{0}=\delta_{1}=\cdots=\delta_{k-1}=0$ and $\delta_{k} \neq 0$.

We can rewrite the $\delta_{i}$ by using more intrinsic notions as follows. Let $c_{*}^{C M}(V)=$ $\sum_{j=0}^{d} c_{j}^{C M}(V)$ be the total Chern-Mather class of $V$. Recall that if $V$ is smooth, $c_{*}^{C M}(V)$ coincides with the usual total Chern class of $V$. Then by (the proof of) [14, Theorem 3.1], we have

$$
\delta_{i}=(-1)^{d+i} \sum_{j=0}^{i}\left(\begin{array}{c}
i+2 \\
j
\end{array}\right)(i+1-j) \int_{V} \frac{h^{j}}{(1+h)^{i+2}} c_{*}^{C M}(V) .
$$

This last formula is very useful for the exact computation of the $\delta_{i}$. Indeed, in [14 we explicitly calculated the degrees of duals of singular varieties in various situations. We thus obtained an effective method to determine the dimensions of the duals of singular varieties, which is similar to Holme's theorem [7, Theorem 3.4 (i)] (see also Beltrametti-Fania-Sommese [1, Gelfand-Kapranov [5] and Tevelev [18, Chapter 6] for related important results for smooth varieties).

Next consider the general case where $X=\mathbb{P}_{n}$ and $Y=\mathbb{G}_{n, k}(0 \leq k \leq n-1)$. Then the $k$-dual $V^{\langle k\rangle} \subset Y=\mathbb{G}_{n, k}$ that we consider here is the one studied by Gelfand-Kapranov-Zelevinsky [6] and Ernström [3]. In [6, Chapter 3, Proposition 2.1 , they showed that if $V^{\langle k\rangle}$ is a hypersurface of $\mathbb{G}_{n, k}$, then we can define the degree of $V^{\langle k\rangle}$ to be that of the defining polynomial of $V^{\langle k\rangle}$ in the Plücker coordinates: $V^{\langle k\rangle} \subset \mathbb{G}_{n, k} \subset \mathbb{P}_{\left(\begin{array}{c}n+1 \\ k+1\end{array}\right)-1}$. In [6, Chapter 3, Proposition 2.2], they also proved that this degree of $V^{\langle k\rangle}$ in $\mathbb{G}_{n, k}$ has a topological interpretation as follows. Let us take linear subspaces $\widehat{L_{1}}, \widehat{L_{2}} \subset X=\mathbb{P}_{n}$ of dimensions $k-1$ and $k+1$ respectively such that $\widehat{L_{1}} \subset \widehat{L_{2}}$ and the pencil

$$
P\left(\widehat{L_{1}}, \widehat{L_{2}}\right)=\left\{\widehat{L} \in \mathbb{G}_{n, k} \mid \widehat{L_{1}} \subset \widehat{L} \subset \widehat{L_{2}}\right\} \simeq \mathbb{P}_{1}
$$

satisfies the conditions

(i) $P\left(\widehat{L_{1}}, \widehat{L_{2}}\right) \cap V_{\text {sing }}^{\langle k\rangle}=\emptyset$,

(ii) $P\left(\widehat{L_{1}}, \widehat{L_{2}}\right)$ intersects with $V_{\text {reg }}^{\langle k\rangle}$ transversally at finite points.

Then the degree of $V^{\langle k\rangle}$ in $\mathbb{G}_{n, k}$ is also given by

$$
\operatorname{deg} V^{\langle k\rangle}=\sharp\left(P\left(\widehat{L_{1}}, \widehat{L_{2}}\right) \cap V^{\langle k\rangle}\right) .
$$

With this topological description of $\operatorname{deg} V^{\langle k\rangle}$ at hand, we can now prove the following theorem.

Theorem 3.4. Let $V \subset X=\mathbb{P}_{n}$ be a projective variety of dimension d. Assume that the $k$-dual $V^{\langle k\rangle} \subset Y=\mathbb{G}_{n, k}$ is a hypersurface. Then we have

$$
\operatorname{deg} V^{\langle k\rangle}=(-1)^{(n-k)+d+1}\left\{\int_{\widehat{M}} \mathrm{Eu}_{V}-2 \int_{\widehat{L}} \mathrm{Eu}_{V}+\int_{\widehat{N}} \mathrm{Eu}_{V}\right\},
$$


where $\widehat{M}, \widehat{L}, \widehat{N} \subset X=\mathbb{P}_{n}$ are generic linear subspaces of dimensions $k-1, k, k+1$, respectively.

Proof. By Theorem 2.8, for a generic linear subspace $\widehat{L} \simeq \mathbb{P}_{k} \subset X$ of dimension $k$ we have

$$
\mathcal{R}_{S}\left(\mathrm{Eu}_{V}\right)=\left(\int_{\widehat{L}} \mathrm{Eu}_{V}\right) \mathbf{1}_{Y}+(-1)^{k(n-k)+\operatorname{dim} V+\operatorname{dim} V^{\langle k\rangle}} \mathrm{Eu}_{V\langle k\rangle} .
$$

Since we assumed that $\operatorname{dim} V=d$ and $\operatorname{dim} V^{\langle k\rangle}=\operatorname{dim} Y-1=(k+1)(n-k)-1$, we get

$$
\mathcal{R}_{S}\left(\mathrm{Eu}_{V}\right)=\left(\int_{\widehat{L}} \mathrm{Eu}_{V}\right) \mathbf{1}_{Y}+(-1)^{(n-k)+d+1} \mathrm{Eu}_{V^{\langle k\rangle}} .
$$

Also for linear subspaces $\widehat{L_{1}}, \widehat{L_{2}} \subset X=\mathbb{P}_{n}$ satisfying the conditions as in (3.11), we obtain

$$
\int_{Y} \mathcal{R}_{S}\left(\mathrm{Eu}_{V}\right) \cdot \mathbf{1}_{P\left(\widehat{L_{1}}, \widehat{L_{2}}\right)}=2 \int_{\widehat{L}} \operatorname{Eu}_{V}+(-1)^{(n-k)+d+1} \operatorname{deg} V^{\langle k\rangle} .
$$

On the other hand, it follows from $\mathcal{R}_{S}^{\vee}\left(\mathbf{1}_{P\left(\widehat{L_{1}}, \widehat{L_{2}}\right)}\right)=\mathbf{1}_{\widehat{L_{1}}}+\mathbf{1}_{\widehat{L_{2}}}$ that we have

$$
\int_{X}\left(\mathrm{Eu}_{V} \cdot \mathcal{R}_{S}^{\vee}\left(\mathbf{1}_{P\left(\widehat{L_{1}}, \widehat{L_{2}}\right)}\right)\right)=\int_{\widehat{L_{1}}} \mathrm{Eu}_{V}+\int_{\widehat{L_{2}}} \mathrm{Eu}_{V}
$$

Therefore by Lemma 2.6, we obtain (3.12) after replacing $\widehat{L_{1}}, \widehat{L_{2}}$ with $\widehat{M}, \widehat{N}$, respectively.

Corollary 3.5. Let $\widehat{L} \simeq \mathbb{P}_{k+1}$ be a generic $(k+1)$-dimensional linear subspace of $\mathbb{P}_{n}$ and consider the usual dual variety $(V \cap \widehat{L})^{*} \subset \mathbb{P}_{k+1}^{*}$ of $V \cap \widehat{L} \subset \widehat{L} \simeq \mathbb{P}_{k+1}$. Then we have

$$
\operatorname{deg} V^{\langle k\rangle}=\operatorname{deg}(V \cap \widehat{L})^{*}
$$

\section{REFERENCES}

[1] M. C. Beltrametti, M. L. Fania and A. J. Sommese, On the discriminant variety of a projective manifold, Forum Math. 4 (1992), no. 6, pp. 529-547. MR.1189013 (93k:14049)

[2] J. L. Brylinski, Transformations canoniques, dualité projective, théorie de Lefschetz, transformations de Fourier et sommes trigonométriques. Géométrie et analyse microlocales, Astérisque 140-141 (1986), pp. 3-134. MR864073 (88j:32013)

[3] L. Ernström, Topological Radon transforms and the local Euler obstruction, Duke Math. J. 76 (1994), pp. 1-21. MR1301184 (96h:32053)

[4] L. Ernström, A Plücker formula for singular projective varieties, Communications in Algebra 25 (1997), pp. 2897-2901. MR.1458736 (98b:14040)

[5] I. M. Gelfand and M. M. Kapranov, On the dimension and degree of the projective dual variety: a $q$-analog of the Katz-Kleiman formula, Gelfand Mathematical Seminars 1990-1992, Birkhäuser, 1993, pp. 27-33. MR.1247282 (94m:14071)

[6] I. M. Gelfand, M. M. Kapranov and A. V. Zelevinsky, Discriminants, resultants and multidimensional determinants, Birkhäuser, 1994. MR1264417 (95e:14045)

[7] A. Holme, The geometric and numerical properties of duality in projective algebraic geometry, Manuscripta Math. 61 (1988), no. 2, pp. 212-256. MR.943533(89k:14093)

[8] M. Kashiwara, Systems of microdifferential equations, Progress in Mathematics 34, Birkhäuser, Boston, 1983. MR725502 (86b:58113)

[9] M. Kashiwara and P. Schapira, Sheaves on manifolds, Grundlehren Math. Wiss. 292, SpringerVerlag, Berlin-Heidelberg-New York, 1990. MR1074006 (92a:58132)

[10] S. L. Kleiman, The enumerative theory of singularities, Real and complex singularities, Sijthoff and Nordhoff International Publishers, Alphen an den Rijn (1977), pp. 297-396. $\operatorname{MR} 0568897(58: 27960)$ 
[11] R. MacPherson, Chern classes for singular varieties, Ann. of Math. 100 (1974), pp. 423-432. MR0361141 (50:13587)

[12] Y. Matsui, Radon transforms of constructible functions on Grassmann manifolds, Publ. Res. Inst. Math. Sci. 42 (2006), no. 2, pp. 551-580. MR2250073 (2007h:32030)

[13] Y. Matsui and K. Takeuchi, Microlocal study of topological Radon transforms and real projective duality, Adv. in Math. 212 (2007), no. 1, pp. 191-224. MR2319767

[14] Y. Matsui and K. Takeuchi, Generalized Plücker-Teissier-Kleiman formulas for varieties with arbitrary dual defect, Proceedings of Australian-Japanese workshop on real and complex singularities, World Scientific, 2007, pp. 248-270.

[15] Y. Matsui and K. Takeuchi, Projective duality and topological X-ray Radon transforms, in preparation.

[16] A. Parusinski, Multiplicity of the dual variety, Bull. London Math. Soc. 23 (1991), pp. 429436. MR1141011 (93a:14006)

[17] P. Schapira, Tomography of constructible functions, Lecture Notes Computer Science 948, Springer, Berlin (1995), pp. 427-435. MR1448182(98e:32056)

[18] E. Tevelev, Projective duality and homogeneous spaces, Encyclopaedia of Mathematical Sciences 133, Springer, 2005. MR2113135 (2005m:14001)

[19] O. Y. Viro, Some integral calculus based on Euler characteristics, Lecture Notes in Math. 1346, Springer-Verlag, Berlin (1988), pp. 127-138. MR970076 (90a:57029)

[20] C. T. C. Wall, Singular points of plane curves, London Math. Soc. Student Texts 63, Cambridge Univ. Press, 2004. MR2107253 (2005i:14031)

Graduate School of Mathematical Sciences, The University of Tokyo, 3-8-1, Komaba, MEguro-KU, TOKYO, 153-8914, JAPAN

E-mail address: you317@ms.u-tokyo.ac.jp

Institute of Mathematics, University of Tsukuba, 1-1-1, Tennodai, Tsukuba, Ibaraki, 305-8571, JAPAN

E-mail address: takemicro@nifty.com 